\title{
The Compression of Gases in Presence of Condensation Centers in Compressible Volume, the Equation of State and Isotherm of Polymolecular Adsorption for the Studied System
}

\author{
A. G. Zakharov \\ Im Neuwerk 5, 21680 Stade, Germany \\ Email: sacharowneuwerk@yahoo.de
}

\begin{abstract}
This work investigates the compression of gases in a closed volume with presence of condensation centers in it. An equation describing the output molecules from the gas phase when it is compressed was suggested. The solution to this equation predicted the existence of two regimes in the development of this process and the possibility of transition from one to the other at a point. We analyzed the effect of the number of condensation centers on the view of the proposed equation, and, consequently, on the process of removal of molecules from the gas phase. Using the obtained results it was proposed the equation of state of real gases, which physically correctly describes their compression and condensation on the active centers. Applied approach to the analysis of the studied system allowed a logically consistent way to obtain the isotherm of polymolecular adsorption, containing the main elements of the experimental curves and a different more simple form from known equations.
\end{abstract}

Keywords: Compression of gases, condensation centers, equation of state, the isotherm of polymolecular adsorption

\section{Introduction}

Heterogeneous processes involving gas phase, include a wide range of industrially important reactions, which causes a constant desire to understand the characteristics of their occurrence. When describing the behaviour of the gas phase in such processes typically use so-called state equation, the simplest of which is the equation of state of an ideal gas:

$$
P V=N k_{B} T
$$

where $\mathrm{P}$ is the gas pressure, $\mathrm{V}$ is volume, $\mathrm{N}$ is the total number of gas molecules in the system, $k_{B}$ is Boltzmann constant, $\mathrm{T}$ is the temperature. An ideal gas is gas in conditions where is permissible to neglect the size of its molecules and the presence of interaction between them. If that neglect is unjustified and to correctly describe the behaviour of gas is required to take account of these factors, the gas is called real. The most well known equation of state of real gases, proposed in 1873, is the equation of van der Waals $[1,2]$

$$
\left(P+\frac{a N^{2}}{V^{2}}\right)(V-N b)=N k_{B} T
$$

where, according to the common interpretation, the second term in the first parenthesis reflects the additional pressure that exists in the system due to the presence of attractive forces between gas molecules ( $a-\mathrm{a}$ dimensional constant), and the second term in the second bracket is equal to the volume that is inaccessible to their movement. Proceeding from the latter, the parameter $b$ can be interpreted as the effective volume of the gas molecules.

Several other equations of state of real gases are known [3-5]. They are applicable to certain specific systems. One of these is the Benedict - Webb - Rubin equation, which particularly well describes the compression of light hydrocarbons and their mixtures. However, to clarify the physical picture of this process, they do not provide additional information compared with the equation of van der Waals, which allows us in this paper to limit the discussion of the latter. 
The task that was solved in the formulation of equation (2), was to describe with a single equation of the process gas compression, his condensation and compression of the liquid formed as a result of condensation. It is not surprising that, because of the complexity of this problem, the mutual influence of the processes under consideration, the van der Waals equation has a number of problems. As shown in [6] these include: 1 . Its effectiveness lies to the right of straight $V=N b$, i.e. the equation, because their structural features does not and cannot describe the compression of liquid; 2. The equation does not describe regions of the simultaneous existence of the gas and liquid phase, i.e. condensation of gas. On its graphical representation there are no areas of constant pressure with changing volume; 3. For certain physically plausible conditions of conducting the process of compression, it predicts pressure transition in a negative region, which cannot be justified.

The solution to these problems is given in [6-8], where the author obtained dependences describing the output of molecules from the gas phase, naturally solving the problem of transition at the point with hyperbolic plot of gas compression at the site of its condensation with constant pressure with changing volume. It also investigated the influence of the size of gas molecules on the process of their removal from the gas phase and obtained the equation of state, a graphical representation of which matches qualitatively well with experimentally recorded curves.

While in $[7,8]$ in a fairly complete form solved the problem of the description of the compression of the gas to the accepted system (ideal gas) in them is not discussed experimentally known fact of the influence of solid impurities (condensation centres) in the gas phase on the process of condensation, and, of course, the equations of state do not contain such parameters as the number of condensation centres $\left(N_{c}\right)$. This allows us to set the work task of the study of peculiarities of the process of compression a chemically inert gases in the presence of condensation centres (solid particles, dust) in the compressible volume with identifying features of their filling by adsorbed gas and obtaining a respective equations of state.

\section{Methodology Compression}

To solve this problem we use the method of reasoning and analysis suggested in $[7,8]$. In these works it is assumed that in a closed volume $V$ is the total number of gas molecules $N$. Part of them is in the form of single particle - we denote them using $N_{g}$ and we assume their as gas phase. All the remaining molecules are associated in complexes with the number of particles of 2 or more representing the microparticles of the liquid. Reducing the number of single molecules in the gas phase can go through double collisions among themselves with the formation of associated complexes consisting of two molecules, and as a result of their adsorption on existing particles of the fluid. The rise is due to the desorption of single molecules from the composition of the associated complexes (micro-particles of the liquid). It is accepted that due to the smallness of the last each of their constituent molecules may participate in the acts of adsorption and desorption.

With that said, the condition of equality of the speeds of increase and decrease in $N_{g}$, will have the following form $[6,7]$

$$
\frac{\alpha_{1} N_{g}^{2}}{V}+\frac{\alpha_{2}\left(N-N_{g}\right) N_{g}}{V}=\beta\left(N-N_{g}\right)
$$

where $\alpha_{1}$ is the rate constant of formation of the associated complex (micro-particles of liquid) resulting from double collisions of gas molecules with each other, $\alpha_{2}$ is the rate constant of the process of their adsorption on the liquid phase, $\beta$ is the rate constant of the desorption process of gas molecules from the micro-particles of the liquid. The dimension of $\alpha_{1}$ and $\alpha_{2}$ is equal to $\left[\mathrm{m}^{3} \cdot \mathrm{h}^{-1}\right]$, and the dimension of $\beta\left[\mathrm{h}^{-1}\right]$.

If both parts of equation (3) are divided by $\beta$ and are denoted by

$$
k_{\mathrm{a} 1}=\frac{\alpha_{1}}{\beta}, k_{\mathrm{a} 2}=\frac{\alpha_{2}}{\beta}
$$


the number of constants in it will be reduced to two, and let them be the equilibrium constants of the relevant processes. The equation (3) will take the following form

$$
\frac{k_{\mathrm{a} 1} N_{g}^{2}}{V}+\frac{k_{\mathrm{a} 2}\left(N-N_{g}\right) N_{g}}{V}=N-N_{g} \cdot .
$$

Physically meaningful solution of this square relative to $N_{g}$ equations can be represented in the form

$$
N_{g}=\frac{V+N k_{\mathrm{a} 2}-\sqrt{4 N V k_{\mathrm{a} 1}+\left(V-N k_{\mathrm{a} 2}\right)^{2}}}{2\left(k_{\mathrm{a} 2}-k_{\mathrm{a} 1}\right)}
$$

Curves calculated by (6), show that this equation for certain values of its constants, satisfactorily describes the main experimentally known features of the process of removal of molecules from the gas phase when it is compressed (Fig.1, curve 1). If the volume of gas is large, all the molecules are in the form of single particles and $N_{g}=N$ (section $\mathrm{BC}$ of the curve 1). When the gas is compressed at point B the pressure reaches its saturation and further $N_{g}$ linearly decreases with decreasing $V$ (plot 0B). The increase in the constant $k_{\mathrm{a} 2}$ shifts the position of a point $\mathrm{B}$ in the region of large volumes, and decreasing this setting brings it closer to the axis of $N_{q}$. The decrease in the total number of molecules in the system also, in accordance with the physical picture of the process, lowers the area $\mathrm{BC}$ of the discussed dependence. Taken upon receipt of the curve 1 Fig. 1 the value of a constant $k_{a 1}$ is significantly less than the value of $k_{\mathrm{a} 2}$. Only under this condition the transition from the field of constancy $N_{g}$ to its linear decrease occurs at the point or almost point. The latter will occur when $k_{\mathrm{a} 1}=0$. If the value $k_{\mathrm{a} 1}$ is near to $k_{\mathrm{a} 2}$, the kink at point B is smoothed, reducing $N_{g}$ starts to this point, and this reduction increases with increasing $k_{\mathrm{a} 1}$ (Fig. 1 curves 2 and 3). Physically this means that the saturation pressure as a characteristic of the system, as a border, peculiar to it, will take place only if the rate of formation fluid (microparticles) resulting from double collisions of the gas molecules will be low. If this condition is not met, then the formation fluid will be continuous, monotonic process at all levels of the system.

\section{$3 \quad$ Hidden Break Point}

This analysis, carried out in $[7,8]$, here can be supplemented by the following considerations. It is obvious that the point $\mathrm{B}$ on the curve 1 in Fig. 1 is a special point of the investigated functional dependence. There is no doubt that this particular point is also on curves 2 and 3 of the same figure, although based on graphical representation, it cannot be assumed that the presence of such features will be described in their process. Naturally, there is a need to find a method to check any functional dependence on the presence of such a singular point. This is not the point of maximum, not the minimum point and not the inflection point of the function. It will probably be consistent to call it a hidden break point of the corresponding functional dependence, thus pointing to the interesting feature that is present in her and in her monotonous character, if it has a similar point.

If you take the derivative of expression (6), in the case of curve 1 of Fig. 1 it is clear that the rate of change of the value of this derivative will have an extremum at the point $B$. The existence of an extremum of the rate of change of the derivative of a function is equivalent to the statement about existence of extremum of the second derivative of this function. The correctness of this hypothesis for this case is easy to verify if we take the second derivative of expression (6) with respect to the variable V. Graphical representation of the resulting dependencies, using data curves 1 and 2 in Fig.1, will have respectively the shape of curves 4 and 5 of the same figure. It is clear the presence of extrema on these dependences, the increase $k_{\text {a1 }}$ leads to the dilution curve, the extremum is less pronounced, but it's there (Fig. 1, curve 5). 


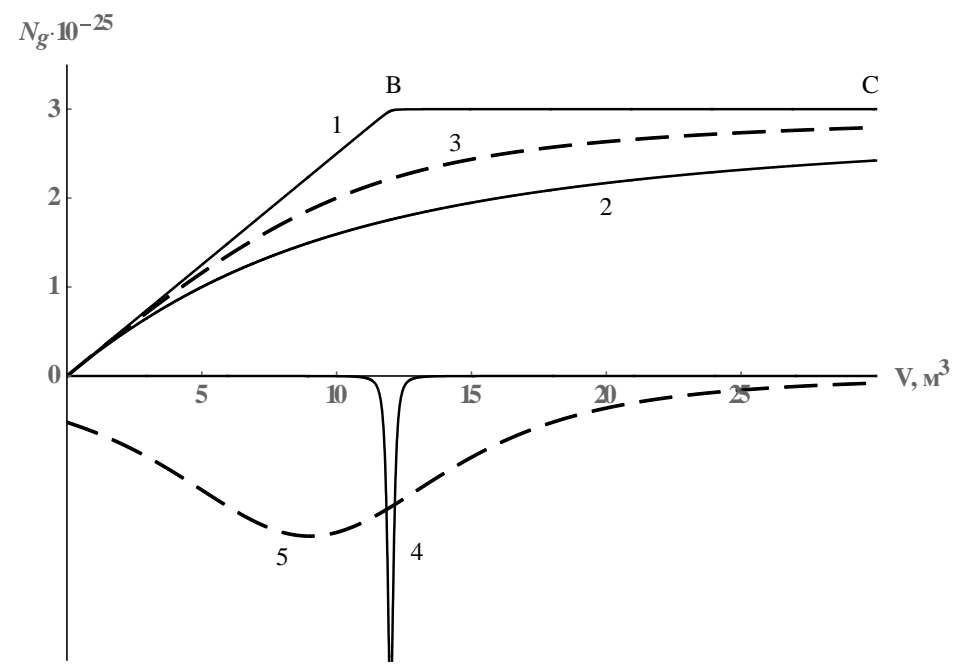

Figure 1. The dependence of the number of molecules in the gas phase from the volume if possible the process of condensation (curves 1,2,3) and second derivatives from functions 1 and 2 (curves 4 and 5 ). All the curves are calculated by $1-N=3 \cdot 10^{-25}, k_{\mathrm{a} 1}=2 \cdot 10^{-29}, k_{\mathrm{a} 2}=4 \cdot 10^{-25} ; \quad 2-N=3 \cdot 10^{-25}, k_{a 1}=5 \cdot 10^{-26}, k_{a 2}=4 \cdot 10^{-25}$; $3-N=3 \cdot 10^{-25}, k_{\mathrm{a} 1}=2 \cdot 10^{-25}, k_{\mathrm{a} 2}=4 \cdot 10^{-25}$.

It should, apparently, be noted that the Langmuir isotherm, although its graphic representation coincides in form with curves 2 and 3 in Fig.1, doesn't have a hidden break point and under no values of the member constants can be transformed to the form of curve 1 of this figure.

You can find which volume corresponds to the point source dependence in the extremes of its second derivative. Clearly, in this case, the value of the third derivative according to (6) will be zero. Doing the proper conversions, it can be shown that this will only be done if:

$$
V=N\left(k_{\mathrm{a} 2}-2 k_{\mathrm{a} 1}\right)
$$

Substituting in the last expression the values $k_{\mathrm{a} 1}, k_{\mathrm{a} 2}$ and $N$ taken upon receipt of the curve 1 in Fig. 1, we obtain that the point $\mathrm{B}$ on the curve, i.e. the break point of the functional dependency corresponding to $V$ is equal to $11.98 \mathrm{~m}^{3}$. On the curve 3 this point is also present and its coordinate is $9 \mathrm{~m}^{3}$.

If we accept that $k_{\mathrm{a} 1}$ in (7) is very small, the corresponding fracture functional dependence will take place at the point, and up to this point and it can be considered that $N_{g}=N$. Then taking into account $(7)$ to determine the saturation pressure $\left(P_{0}\right)$, which as shown in [7], as well

$$
P_{0}=\frac{k_{B} T}{k_{\mathrm{a} 2}}
$$

i.e. it is determined by two constants and temperature, and one of the constants is introduced in $[6,7]$ constant $k_{\mathrm{a} 2}$.

It is clear that this analysis defines a new class of physically important functions, with hidden special points (hidden break point of the function) and one is able to make this point clear by a corresponding change in the conditions of flow describing their process. It is also clear that the study of the properties of this class of functions should be the task of a separate research.

\section{Compression with Condensation Centres}

Having this approach and the results obtained with its help, one can proceed to an analysis of those features of the process that will be due to the presence of condensation centres in the compressible volume. Let us assume that in a closed volume is the total number of gas molecules $N$ and the 
adsorption centres $N_{c}$. Part of the gas molecules is in the gas phase as single particles $N_{g}$, and some in the adsorbed state on adsorption centres $N-N_{g}$. Reduction of the molecules in the gas phase will go through their adsorption on the vacant centres $N_{c}$, and in the adsorbed state the number of molecules of the gas phase, as noted above, is $N-N_{g}$. Desorption rate of molecules from the surface will be proportional to the quantity adsorbed on it, i.e. the same $N-N_{g}$. If, as the simplest assumption to make that all adsorbed molecules are equally accessible to adsorption-desorption processes proceeding with their participation, for the case of equilibrium can be written

$$
\frac{k_{\text {a1 }} N_{g}\left(N_{c}-N+N_{g}\right)}{V}+\frac{k_{\text {a2 }}\left(N-N_{g}\right) N_{g}}{V}=N-N_{g}
$$

where $k_{\mathrm{a} 1}$ and $k_{\mathrm{a} 2}$ - the equilibrium constant for the adsorption process at adsorption centres and the already adsorbed molecules of the gas phase. Equation (9) solves the same task as equation (5), i.e. it describes the change in the number of molecules in the gas phase when it is compressed, but for the case of the presence in the compressible volume of the condensation centres $N_{c}$. Its physically meaningful solution can be represented in the form

$$
N_{g}=\frac{V-N k_{\mathrm{a} 1}+N k_{\mathrm{a} 2}+k_{\mathrm{a} 1} N_{c}-\sqrt{4 N V\left(k_{\mathrm{a} 1}-k_{\mathrm{a} 2}\right)+\left(V+N k_{\mathrm{a} 2}+k_{\mathrm{a} 1}\left(-N+N_{c}\right)\right)^{2}}}{2\left(-k_{\mathrm{a} 1}+k_{\mathrm{a} 2}\right)}
$$

Last equation, as and equation (6), predicts the existence of two possible types of regularities of output molecules from the gas phase (Fig. 2).

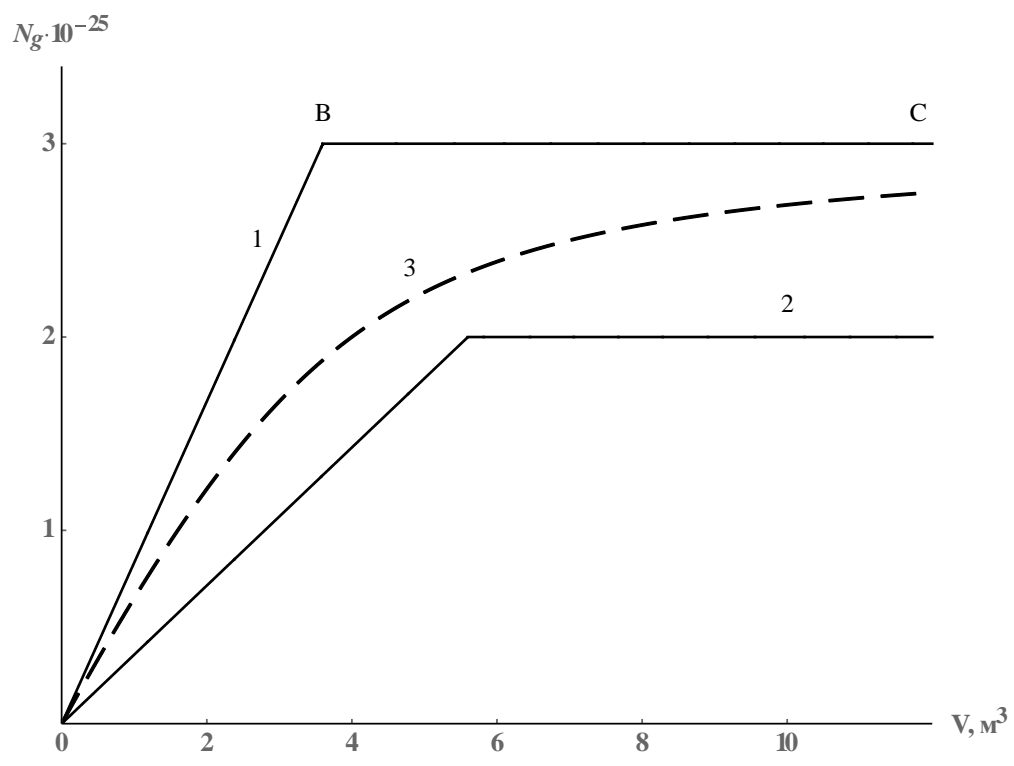

Figure 2. The dependence of the number of molecules in the gas phase from $\mathrm{V}$ in the presence of the condensation centers in the compressible volume. All the curves are calculated by (10) with:

$$
\begin{aligned}
& 1-N=3 \cdot 10^{-25}, N_{c}=8 \cdot 10^{13}, k_{\mathrm{a} 1}=1 \cdot 10^{-25}, k_{\mathrm{a} 2}=2.2 \cdot 10^{-25} ; \\
& 2-N=3 \cdot 10^{-25}, N_{c}=1 \cdot 10^{13}, k_{\mathrm{a} 1}=1 \cdot 10^{-25}, k_{\mathrm{a} 2}=3.8 \cdot 10^{-25} ; \\
& 3-N=3 \cdot 10^{-25}, N_{c}=8 \cdot 10^{24}, k_{\mathrm{a} 1}=1 \cdot 10^{-25}, k_{\mathrm{a} 2}=2.2 \cdot 10^{-25} .
\end{aligned}
$$

In one case, the number of molecules in the gas remains constant up to a certain volume, and then linearly decreases with its decrease (Fig. 2, curves 1 and 2), another same - output curve of molecules of gas has no singular points and notes $N_{g}$ decrease with decreasing $V$ in any field of its values (Fig. 2, curve 3). Dependencies calculated by (10), have the same features as the results obtained when 
calculating according to the equation (6). Curves 1 and 2 in Fig. 2 show that if the volume of gas is large, all the molecules are in the form of single particles and $N_{g}=N$ (section BC of the curve 1). When the gas is compressed at point B the pressure is reached to its saturation and further $N_{g}$ linearly decreases with decreasing $V$ (plot $0 \mathrm{~B}$ ). The increase in the constant $k_{\mathrm{a} 2}$ shifts, as in the previous case, the position of a point $\mathrm{B}$ in the region of large volumes, and decreasing this setting brings it closer to the axis $N_{g}$. The decrease in the total number of molecules in the system also, in accordance with the physical painting process, lowers the sun discussing plot dependence (Fig. 2, curve 2).

Unlike equation (6) equation (10) allows following $N_{c}$ influence on the patterns of output molecules from the gas phase when it is compressed. Curve 3 in Fig. 2 obtained for the same values of all permanent and curve 1 , except $N_{c}$, which is taken equal to $10^{24}$. It is seen that the increase in the number of active centres in the volume led to a qualitative change in the patterns of output molecules from the gas phase when it is compressed. The process has become monotonous character - on the curve 3 there is no point that separates the regions of saturated and unsaturated gas.

On the basis of equation (10) one can directly trace the dependence of the number of molecules in the gas phase $\left(N_{g}\right)$ on the number of adsorption centres in the compressible volume $\left(N_{c}\right)$. Four curves obtained by appropriate calculations are shown in Fig. 3.

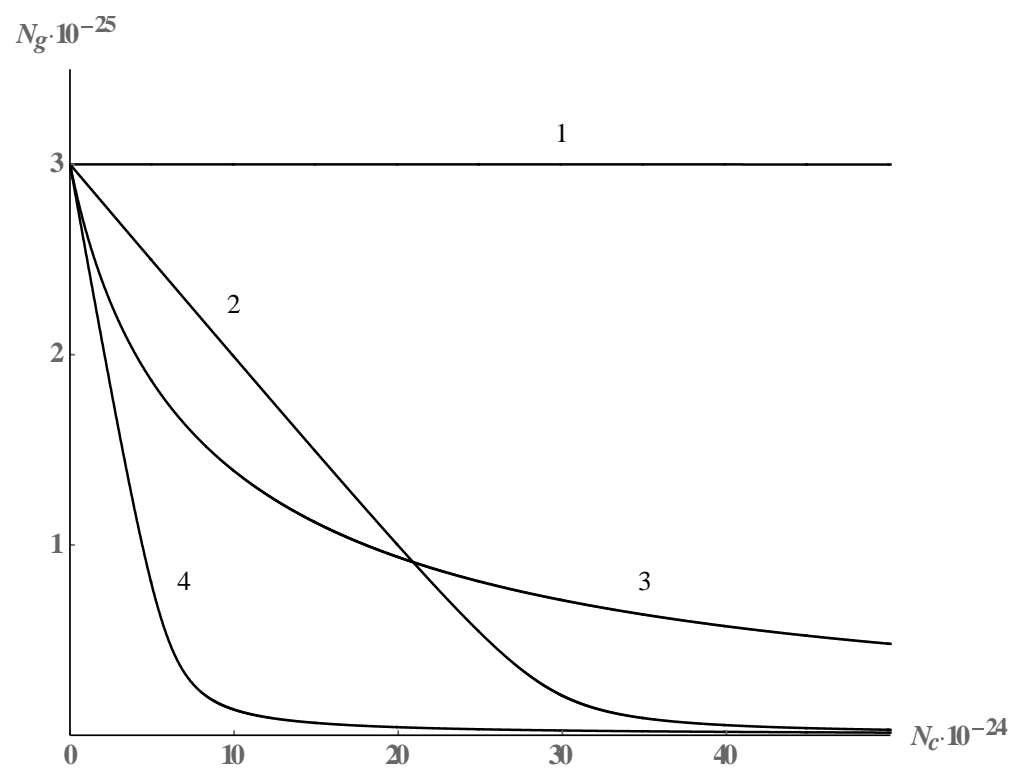

Figure 3. The dependence of the number of molecules in the gas phase from the number of the condensation centers in the compressible volume. All the curves are calculated by (10) with: $N=3 \cdot 10^{-25}, V=1$; $1-k_{\mathrm{a} 1}=5 \cdot 10^{-30}, k_{\mathrm{a} 2}=1 \cdot 10^{-31} \quad ; \quad 2-k_{\mathrm{a} 1}=5 \cdot 10^{-24}, k_{\mathrm{a} 2}=1.1 \cdot 10^{-25} \quad ; \quad 3-k_{a 1}=1 \cdot 10^{-25}, k_{a 2}=1.1 \cdot 10^{-25} \quad ;$ $4-k_{\mathrm{a} 1}=5 \cdot 10^{-24}, k_{\mathrm{a} 2}=4 \cdot 10^{-24}$.

Apparently they reflect physically expected picture of the process. For small $k_{\mathrm{a} 1}$ and $k_{\mathrm{a} 2}$, i.e. at a low intensity of adsorption-desorption processes in the system, introduction to compressible volume of additional number of active centres has virtually no effect on the value of $N_{g}$ (Fig. 3, curve 1). The increase $k_{\mathrm{a} 1}$ and $k_{\mathrm{a} 2}$, leads to the fact that $N_{g}$ decreases with increasing $N_{c}$ (Fig. 3, curve 2). For large values of $k_{\mathrm{a} 1}$ this decrease becomes linear, which suggests that it is caused by the adsorption on the active centres (Fig. 3, curve 2). If you save the values $k_{\mathrm{a} 1}$ to increase $k_{\mathrm{a} 2}, N_{g}$ is reduced in the region of small $N_{c}$ (Fig. 3, curve 3), which, apparently, points to the increasing role of poly molecular 
adsorption under these conditions. Even greater $k_{\mathrm{a} 2}$ leads to even greater reduction in $N_{g}$ at small $N_{c}$, what clearly testifies to the active flow in the case of polymolecular adsorption.

It should also be noted that all the calculated variants of the studied dependence indicate the equality of the number of molecules in the gas phase $\left(N_{g}\right)$ and the total number of molecules in the system $(N)$ if there are no condensation centres in the compressible volume $\left(N_{c}=0\right)$. The same result can be obtained if you take the limit from the right side of equation (10) with $N_{c}$ tending to zero. This limit is equal to $N$. The Implementation of these physically reasonable requirements can, apparently, be regarded as a definite confirmation of the correctness of equation (10).

\section{$5 \quad$ Equation of State}

In the presence of dependence (10) can be solved for the analysed case one of the problems of the equation of Van-der-Waals - task transition at the point with hyperbolic plot of gas compression at the site of its condensation with constant pressure with changing volume. For this we write the equation of state of ideal gases (1) in the form

$$
P V=N_{g} k_{B} T
$$

which taking into account that the number of molecules in the gas phase is not constant and may vary during the compression process. It is clear that in (11) $N_{g}$ shall be determined by (10). Calculated using these two equations the curves are shown in Fig. 4.

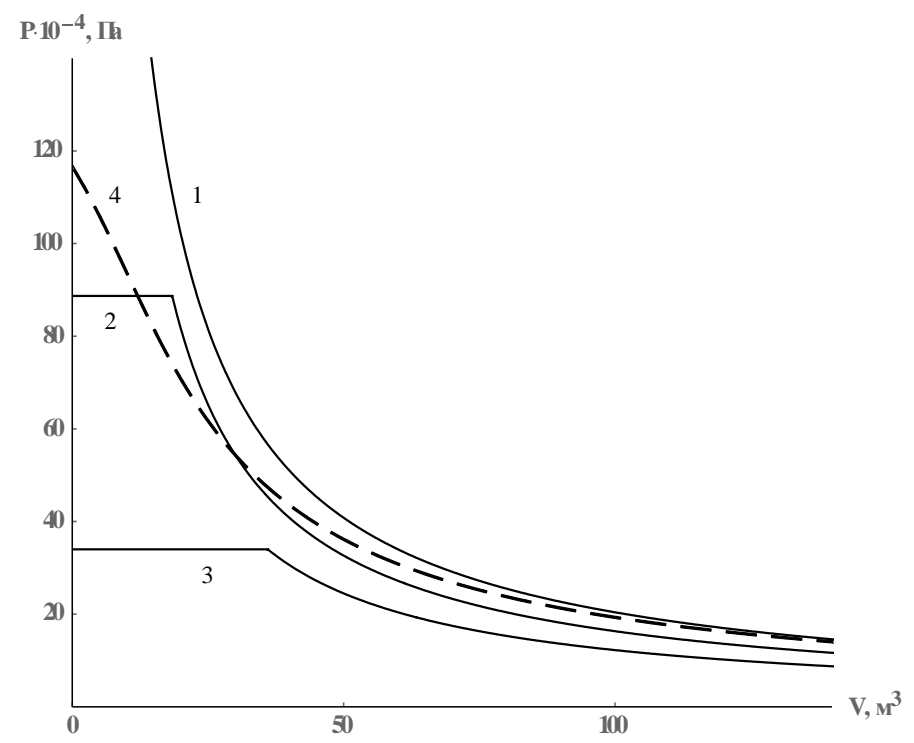

Figure 4. Isotherms of the ideal gas in the presence of the centers of condensation in the compressible volume. Calculated by (11) with (10) with: $k_{B}=1.36 \cdot 10^{-23}, T=300, k_{\mathrm{a} 1}=1 \cdot 10^{-25}$;

$1-N=5 \cdot 10^{25}, N_{c}=1 \cdot 10^{20}, k_{\mathrm{a} 2}=3.5 \cdot 10^{-25} ; 2-N=4 \cdot 10^{25}, N_{c}=1 \cdot 10^{20}, k_{\mathrm{a} 2}=5.6 \cdot 10^{-25}$;

$3-N=3 \cdot 10^{25}, N_{c}=1 \cdot 10^{20}, k_{\mathrm{a} 2}=1.3 \cdot 10^{-24} ; 4-N=5 \cdot 10^{25}, N_{c}=5 \cdot 10^{25}, k_{\mathrm{a} 2}=3.5 \cdot 10^{-25}$.

For greater clarity, they are shifted relative to each other by changing the parameter $N$. Curve 1 (Fig. 4) obtained at low $k_{\mathrm{a} 2}$, qualitatively coincides with the isotherm of the ideal gas (with zero $k_{\mathrm{a} 2}$ this coincidence will be of a quantitative nature). There is no plot of constant pressure with changing volume. The increase of the equilibrium constant of the adsorption process of gas molecules on liquid $k_{\mathrm{a} 2}$ leads to a similar plot to relevant curves (curves 2 and 3), i.e. the isotherm condition begins to reflect the process of condensation of gas. It is clear that with more $k_{\mathrm{a} 2}$ this plot corresponds to a lower 
pressure, which coincides with the result expected from a real process. The transition point of the function with a hyperbolic plot to the plot horizontal line coincides with the break point function constructed by (10) with appropriate values of constants. It is clear that the pressure values on the plot a horizontal line equal to the saturation pressure and must be determined by (8).

When calculating the curve (4) in Fig. 4 all constant values except $N_{c}$, which increased by five orders of magnitude, equal to the corresponding values used to obtain curve 1 . It is seen that the increase in the number of active centres in the compressible volume leads to the decrease in pressure with a corresponding $V$ - curve 4 in the whole area presented in Fig. 4, lies below curve 1. It is clear that such behaviour is physically reasonable. Adsorption of in addition entered centres should lead to the reduction of the pressure in the volume. In addition a large number of active centres in the compressible volume lead to the blurring of the condensation area, on curve 4 there is no plot of constant pressure with changing volume. The same effect can be achieved with curves 2 and 3 , if adopted in the calculations the value $N_{c}$ is increased to $10^{25}$.

The output curves of Fig. 2 to zero correspond to the accepted model of an ideal gas, whose molecules do not occupy any volume. In case of a real gas in equation (9) (as is done in obtaining equation of Van der Waals) can introduce an amendment, taking into account the effective volume of molecules $\left(V_{\mathrm{m}}\right)$ :

$$
\frac{\mathrm{k}_{\mathrm{a} 1} \mathrm{~N}_{\mathrm{g}}\left(\mathrm{N}_{\mathrm{c}}-\mathrm{N}+\mathrm{N}_{\mathrm{g}}\right)}{\mathrm{V}-\mathrm{NV}_{\mathrm{m}}}+\frac{\mathrm{k}_{\mathrm{a} 2}\left(\mathrm{~N}-\mathrm{N}_{\mathrm{g}}\right) \mathrm{N}_{\mathrm{g}}}{\mathrm{V}-\mathrm{NV}_{\mathrm{m}}}=\mathrm{N}-\mathrm{N}_{\mathrm{g}}
$$

The program "Mathematica 10" gives the solution of this equation in the following a little cumbersome, but productive form:

$$
N_{g}=\frac{A+B}{2\left(k_{a 1}-k_{a 2}\right)}
$$

where

$$
A=-N k_{a 2}+k_{a 1}\left(N-N_{c}\right)
$$

$B=\left(V-N V_{m}\right)\left(-1+\sqrt{\frac{k_{a 1}^{2}\left(N-N_{c}\right)^{2}+\left(-V+N k_{a 2}+N V_{m}\right)^{2}-2 k_{a 1}\left[\left(N+N_{c}\right)\left(-V+N V_{m}\right)+N k_{a 2}\left(N-N_{c}\right)\right]}{\left(V-N V_{m}\right)^{2}}}\right)$

The results of calculations based on the latest equation with a relatively small $N_{c}$ (if $N=10^{25}, N_{c}$ is of the order of $10^{15}$ ) give curves 1 and 2 in Fig. 5.

The form of curves is quite unusual. Since they have both a break point for the function and a point for $V=N V_{m}$ of an abrupt change in its value. For all the unusual results of the calculation from the functional dependence (13), the progress of the curves obtained is physically justified. In the region less than $N V_{m}$, the formed liquid with the number of molecules in $\mathrm{N}$ is compressed, and it will not change under compression. This reflects the results of the calculation done by the program "Mathematica 10" (Fig. 5).

If $N_{c}$ is large (approaches the value $\mathrm{N}$ or exceeds) the calculated curves appear distortion get rid of which is possible given the physical constraints imposed on the process, designing the appropriate equation with the introduction of two functions Heaviside (Heaviside) $[9,10]$

$$
\mathrm{He}_{1}=\operatorname{UnitStep}\left[V-N V_{m}\right], \mathrm{He}_{2}=\operatorname{UnitStep}\left[-V+N V_{m}\right]-\operatorname{UnitStep}[-V]
$$

With this in mind, as shown in [4], the expression for $N_{g}$ will be as follows

$$
N_{g}=N_{\mathrm{g} 0} \mathrm{He}_{1}+\mathrm{NHe}_{2}
$$

where $N_{g 0}$ should in discuss case be determined by (13). The expression (15) results also in the large $N_{c}$ to physically meaningful representations (Fig. 5, curve 3). It is seen that the increase in the number of active centres in the compressible volume makes possible the withdrawal of molecules from the gas phase and by large volume of the system, that corresponds to the physical concepts. Of course, using the 
dependencies (15) we can obtain curves 1 and 2 in Fig. 5. In this case, it is interesting that at small $N_{c}$ using the program "Mathematica 10" and without the use of functions Heaviside gets the same results.

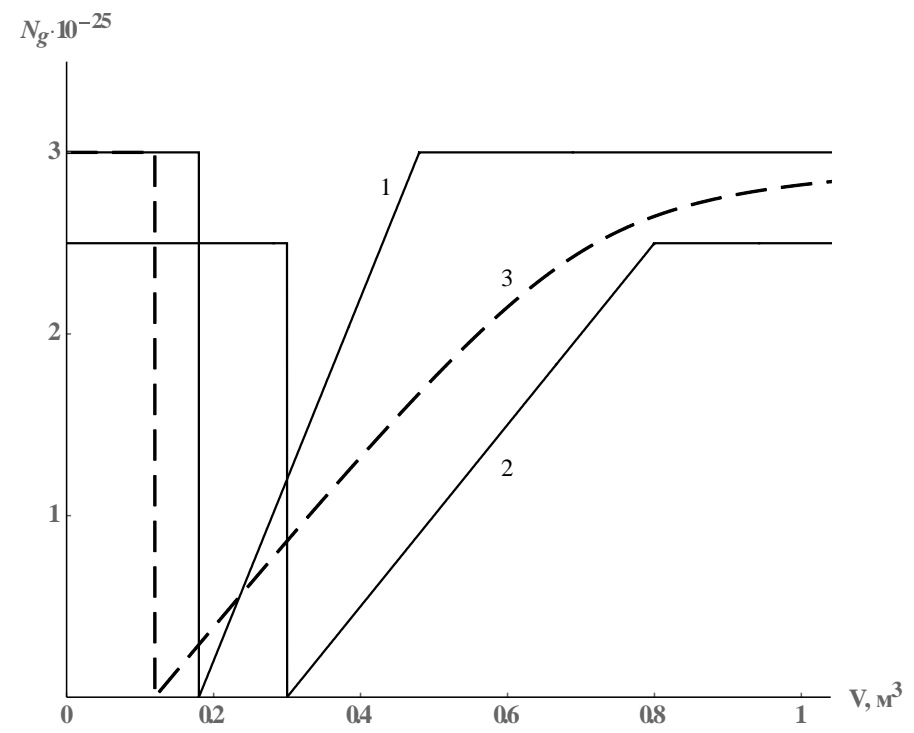

Figure 5. The dependence of the number of molecules in the gas phase from $\mathrm{V}$, the inequality to zero their own volume $V_{m}$. Calculated by (10) with:

$$
\begin{aligned}
& 1-N=3 \cdot 10^{25}, N_{c}=1 \cdot 10^{20}, k_{\mathrm{a} 1}=1 \cdot 10^{-30}, k_{\mathrm{a} 2}=1 \cdot 10^{-26}, V_{m}=6 \cdot 10^{-27} \\
& 2-N=2.5 \cdot 10^{25}, N_{c}=1 \cdot 10^{15}, k_{\mathrm{a} 1}=1 \cdot 10^{-30}, k_{\mathrm{a} 2}=2 \cdot 10^{-26}, V_{m}=12 \cdot 10^{-27} \\
& 3-N=3 \cdot 10^{25}, N_{c}=2 \cdot 10^{28}, k_{\mathrm{a} 1}=1 \cdot 10^{-30}, k_{\mathrm{a} 2}=2 \cdot 10^{-26}, V_{m}=4 \cdot 10^{-27}
\end{aligned}
$$

With equation (13), similar to the way it was done for the case of ideal gas, one can go over to the equation of state that takes into account the size of its molecules. For this purpose, in accordance with van der Waals, we introduce in the equation of state (1) a correction for volume of molecules and, in addition, according to the above, consider the difference between their total number in the system $(\mathrm{N})$ and in the gas phase $\left(N_{g}\right)$, i.e.

$$
P\left(V-N V_{m}\right)=N_{g} k_{B} T
$$

where $N_{g}$ at low $N_{c}$ can be determined by (13), and at large it is necessary to use the dependence (15). Four curves calculated using these dependencies are shown in Fig. 6, the first and second of them differ only in the parameter $k_{\mathrm{a} 2}$, and the third and fourth for greater clarity, they are shifted relatively along the axis $\mathrm{V}$ owing to the change of parameter $\mathrm{N}$ and differ from each other by the value $N_{c}$. It is seen that at small $k_{\mathrm{a} 2}$ (curve 1) corresponding graph is a hyperbola with a vertical asymptote equal to $N V_{m}$ in the region of positive $\mathrm{V}$. Increasing $k_{\mathrm{a} 2}$ transforms the graphical representation in such a way that it appears three phase (curve 2). The first of them, lying in the region of large volumes, consistent with the compression of gas, the second, the pressure of which is constantly changing in volume - the process of its condensation, and the third with a sharp increase in pressure with little change in volume (according to the interpretations adopted in the analysis of the isotherms of van der Waals) can be associated with compression of the liquid. An important, although maybe subtle is the fact that the last plot on the curve with a high degree of accuracy coincides with the position of the border separating the pure liquid region from the region in which is present in the system and the gas phase, i.e. with a line $\mathrm{V}=N V_{m}$. As noted in [7] in the analysis of the van der Waals isotherm, the whole of it, including the region closest to the $\mathrm{P}$ axis, lies to the right of the line $\mathrm{V}=N b$, i.e. the corresponding dependence can not describe the compression of the liquid. 


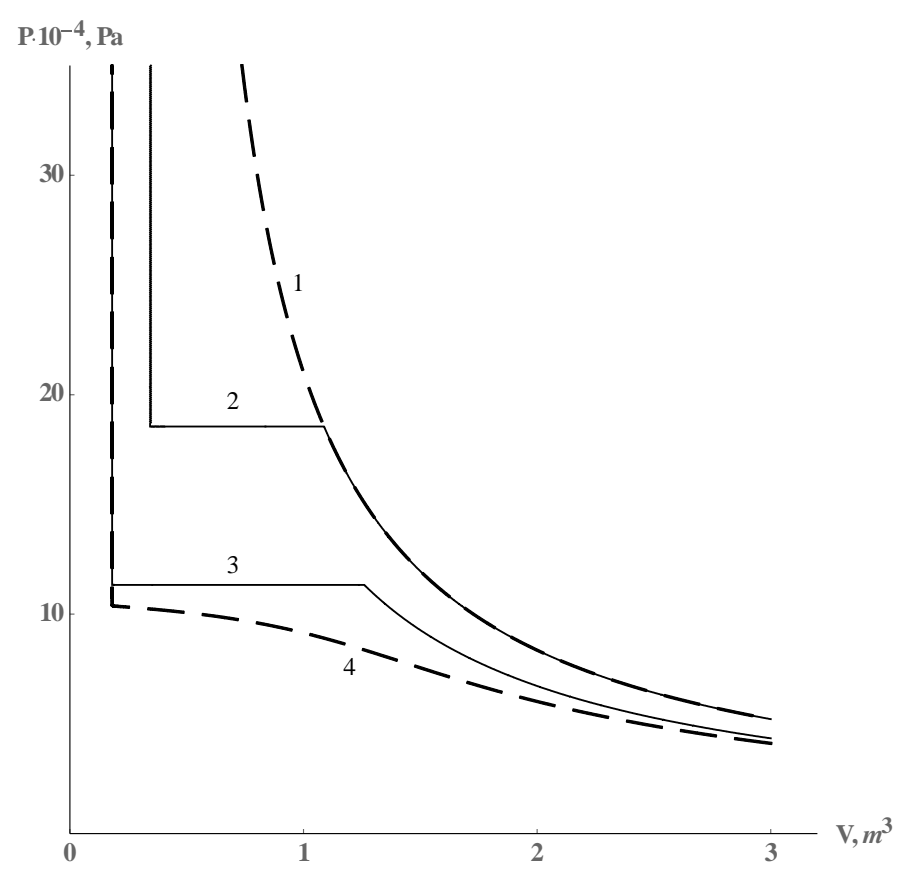

Figure 6. Isotherms of a real gas state in the presence of the centres of condensation in the compressible volume. Calculated by (16) with (15) when:

$$
\begin{aligned}
& 1-N-3,4 \cdot 10^{25}, N_{c}=1 \cdot 10^{15}, k_{\mathrm{a} 1}=1 \cdot 10^{-30}, k_{\mathrm{a} 2}=2 \cdot 10^{-27}, V_{m}=1 \cdot 10^{-26} \\
& 2-N=3,4 \cdot 10^{25}, N_{c}=1 \cdot 10^{15}, k_{\mathrm{a} 1}=1 \cdot 10^{-30}, k_{\mathrm{a} 2}=2,2 \cdot 10^{-26}, V_{m}=1 \cdot 10^{-26} \\
& 3-N=3 \cdot 10^{25}, N_{c}=1 \cdot 10^{20}, k_{\mathrm{a} 1}=1 \cdot 10^{-30}, k_{\mathrm{a} 2}=3,6 \cdot 10^{-26}, V_{m}=6 \cdot 10^{-27} \\
& 4-N=3 \cdot 10^{25}, N_{c}=1 \cdot 10^{29}, k_{\mathrm{a} 1}=1 \cdot 10^{-30}, k_{\mathrm{a} 2}=3,6 \cdot 10^{-26}, V_{m}=6 \cdot 10^{-27}
\end{aligned}
$$

The increase of $k_{\mathrm{a} 2}$, as in the case of Fig. 2, makes lower saturation pressure (curve 3), which obviously coincides with the result expected from a real process. If $N_{c}$ is relatively small, then the curve preserves all the above mentioned elements and it is possible to talk about the concept of saturation pressure (curves 2 and 3 of figure 6). Curve 4 in Figure 6 was obtained for the same values of all constants as curve 3 with the exception of $N_{c}$, which is increased by four orders of magnitude. It is seen that for large $N_{c}$ a process of formation of fluid goes in the whole area of the investigated volume and, as a consequence, the corresponding isotherm (curve 4 of figure 6) has no areas of constant pressure with changing $V$. The output plots for the compression of fluid for curves 3 and 4 coincide and do not depend on the number of condensation centres $\left(N_{c}\right)$ in the compressible volume.

\section{Isotherm of Polymolecular Adsorbtion}

It is clear that the closeness to reality of the obtained results depends on the degree of correctness of the fundamental equation (9). To check its efficiency will analyse the changes in the interpretation of polymolecular adsorption, which leads to the proposed approach to the study of the process of gas compression in the presence of the centres of condensation in the compressible volume. Given that the number of adsorbed molecules $N_{a}$ is determined by the following expression

$$
N_{a}=N-N_{g}
$$

source dependence (9) can be transformed to mind

$$
\frac{k_{\mathrm{a} 2} N_{a} N_{g}}{V}+\frac{k_{\mathrm{a} 1}\left(-N_{a}+N_{c}\right) N_{g}}{V}=N_{a}
$$

From the last equation $N_{a}$, we get 


$$
N_{a}=\frac{k_{\mathrm{a} 1} N_{c} N_{g}}{V+k_{\mathrm{a} 1} N_{g}-k_{\mathrm{a} 2} N_{g}}
$$

If in this equation substitute $V$ from (11), it is converted to an interesting sight:

$$
N_{a}=\frac{k_{\mathrm{a} 1} N_{c} P}{k_{\mathrm{a} 1} P-k_{\mathrm{a} 2} P+k_{B} T}
$$

Equation (20) represents the isotherm of polymolecular adsorption. We see immediately that in the absence of adsorption in the second and subsequent layers, i.e. $k_{\text {a2 }}$ equal to zero, it takes the form of the Langmuir isotherms (Fig. 7, curve 1).

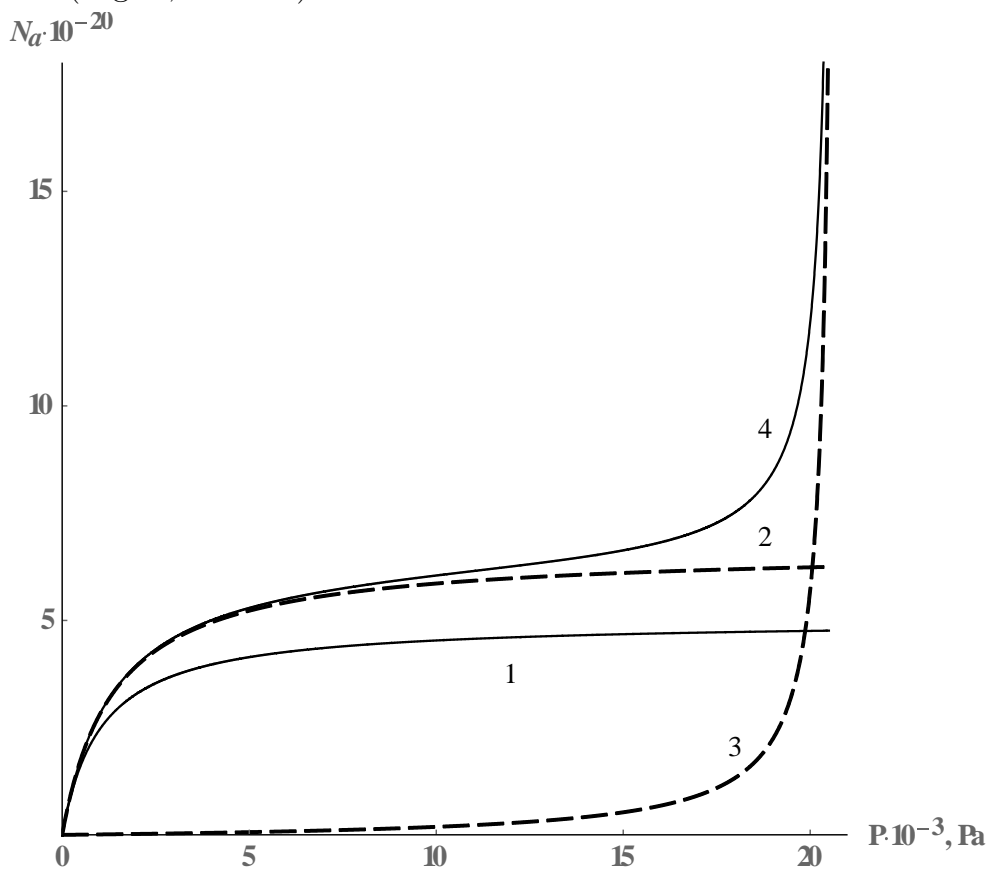

Figure 7. The adsorption isotherm was calculated according to the equation (20) for different values of constituent constants: $T=150, k_{B}=1,38 * 10^{-23} ; 1-N_{c}=5 \cdot 10^{20}, k_{\mathrm{a} 1}=2 \cdot 10^{-24}, k_{\mathrm{a} 2}=0$;

$2-N_{c}=5 \cdot 10^{20}, k_{\mathrm{a} 1}=2 \cdot 10^{-24}, k_{\mathrm{a} 2}=7 \cdot 10^{-25} ; 3-N_{c}=1 \cdot 10^{20}, k_{\mathrm{a} 1}=2 \cdot 10^{-26}, \mathbb{R}_{\mathrm{a} 2}=1,2 \cdot 10^{-25}$

If $k_{\mathrm{a} 2}$ is not zero, it is, of course, can be either less than $k_{\mathrm{a} 1}$ or more. When $k_{\mathrm{a} 2}<k_{\mathrm{a} 1}$ the calculation by the equation (20) gives the results, a graphical representation of which coincides in form with the Langmuir isotherm (Fig. 7, curve 2). More filling is justified, as the curve 2 in Fig. 7 is isotherm of polymolecular adsorption, while the curve 1 of this figure represents the monomolecular adsorption isotherm. Apparently this is a new approach to the problem, because in the literature it is impossible to find statements that the isotherms of monomolecular and poly molecular adsorption can be identical in form. It is clear that in addition to purely cognitive interest of this assertion requires a more careful approach to the use of the adsorption methods for the determination of solid surfaces.

When $k_{\mathrm{a} 2}>k_{\mathrm{a} 1}$ increase in pressure will result in a reduction of the denominator of expression (20), and thus to increase the fill. Thus, from physical considerations, it is clear that the pressure in such a system may not exceed a certain value determined by the requirement of equality to zero of the denominator of expression (20), and represents the saturation pressure $P_{0}$. For its definition we have

$$
P_{0}=\frac{k_{B} T}{k_{\mathrm{a} 2}-k_{\mathrm{a} 1}}
$$

If $k_{\mathrm{a} 1}$ in the latter equation is so small that its value can be neglected, it coincides in form with the dependence (8), obtained for the system not containing in the compressible volume of the centres of condensation. 
The results of the calculation in (20) for the case when $k_{\mathrm{a} 2}>k_{\mathrm{a} 1}$ is curve 3 of figure 7 , as well as the curve 2 , is the isotherm of polymolecular adsorption of a gas on a solid surface. This considerable difference in the shapes of isotherms occurs as a consequence of different relationships between the constants $k_{\mathrm{a} 1}$ and $k_{\mathrm{a} 2}$ for these cases. If we accept that $k_{\mathrm{a} 2}$ is constant, everything begins to depend on the characteristics of the surface $k_{\mathrm{a} 1}$. If the surface is formed by the centres with $k_{\mathrm{a} 1}$ more than $k_{\mathrm{a} 2}$, the isotherm of poly molecular adsorption will be in the form of curve 2 of figure 7 . If it is formed by centres with $k_{\mathrm{a} 1 \text { ? }}$ less than $k_{\mathrm{a} 2}$, the corresponding isotherm will be in the form of a curve 3 of this figure. An interesting situation is when the surface is presented by the centres and the first and second type. It is clear that total filling will be equal to the sum of fill centres of each type. For the cases of curves 2 and 3 figure 7 the summation leads to curve 4 of the same figure. We immediately see that this curve is well reflected by numerous experimental data. Traditionally, the initial portion it is interpreted as the filling of the monolayer and a subsequent increase coverage at higher pressures due to the formation of a second and next adsorbed layer on the surface, i.e. the development of polymolecular adsorption. Based on the foregoing it is clear that such an understanding may not always correspond to objective reality. The shape of the curve 4, for example, in every section depends on polymolecular adsorption. Two mechanisms to increase the fill are explained in the analysis of figure 7 and they are connected with the presence on the surface of two types of centres with different values of the parameter $k_{\mathrm{a} 1}$. The proposed understanding enables changes in a wide range of curve 4 in figure 7 , and, as a consequence, allows to obtain a satisfactory agreement of calculation results with experimental data.

\section{Conclusion}

In conclusion of this work, we can say that the proposed approach to the analysis of the gas compression process in the presence of condensation centres in the compressible volume allowed us to obtain a physically consistent picture of it, explaining, apparently, all experimentally known features of its flow.

1. It is shown for the first time that the investigated process is described by functions having hidden special points, which becomes apparent only under certain conditions of its maintenance.

2. The presence of these points made it possible to solve the old problem of describing transitions from the hyperbolic gas compression region to its condensation section with constant pressure under a varying volume.

3. It is shown that an increase in the number of condensation centres makes the special points hidden, the corresponding functional dependencies become monotonous.

4. Taking into account these results, the equation of state of real gases is proposed, which naturally describes the hyperbolic section of their compression, the region of their condensation with constant pressure at a varying volume, and the compression section of the formed liquid.

5. This analysis made it possible to determine the filling of the condensation centres by adsorbed gas, i.e. obtain isotherms of polymolecular adsorption for the studied system. The latter differs from those known by a simpler form and opens up the possibility of a physically more realistic simulation of the process described by them. The resulting equation of state and isotherm of polymolecular adsorption for the studied system were obtained, leading to a new understanding of the flowing in it processes.

\section{References}

1. Diu B, Guthmann C, Lederer D, Roulet B. Grundlagen der Statistischen Physik. Berlin-N.Y.: Walter de Gruyter, 1994. 1396 S.

2. https://de.wikipedia.org/wiki/Van-der-Waals-Gleichung

3. Reid R, Prausnitz J., Sherwood T. Properties of gases and liquids. L.: Chemistry, 1982. 591 p.

4. M. Benedict, G. B. Webb, L. C. Rubin J.Chem.Phys., 1940, No. 8. S. 334.

5. https://de.wikipedia.org/wiki/Zustandsgleichung_von_Benedict-Webb-Rubin

6. Zakharov A. G. //Solid Fuel Chemistry. 2008. No. 1. S. 46.

7. Zakharov A. G. //Solid Fuel Chemistry. 2008. No.5. S. 29.

8. Zakharov A. G. //Journal of Physical Chemistry. 2011. Vol. 85, No. 5. S. 815. 
9. Matthews, J., Walker, R. Mathematical methods of physics. M.: Atomizdat, 1972. 392 p.

10. https://de.wikipedia.org/wiki/Heaviside-Funktion 